\title{
Consuming nutritional doses of olive pomace oil fulfils recommended dietary allowances of alpha-tocopherol. Results from a randomized clinical trial
}

\section{Abstract}

Vitamin E is a powerful antioxidant that prevents free radical propagation in membranes and plasma lipoproteins. Recommended Daily Allowance (RDA), stablished at 13 and $11 \mathrm{mg} /$ day for adult men and women, respectively, is not fulfilled in most European countries. Thus, strategies to cover the daily recommendations of this vitamin are essential, and one may be to encourage the consumption of foods rich in this micronutrient.

Olive-pomace oil (OPO), which is obtained from the solid by-product obtained during olive oil extraction, is nutritionally relevant due to its high content in oleic acid (C18:1), among other monounsaturated fatty acids. In addition, OPO contains a wide range of minor bioactive components that may contribute to its healthy properties. Among these, it is especially relevant the high content of vitamin E in OPO.

The objective of the present study was to assess the effect of consuming nutritional relevant doses of olive pomace oil within a normal, non-supplemented diet, in the vitamin E status in adults.

We carried out a randomized, cross-over, controlled clinical trial, 14 weeks long in 70 subjects, consisting in two 4-week interventions in which volunteers consumed either OPO or high oleic sunflower oil (HOSO) preceded each by 3-week wash-out steps with sunflower oil. Participants daily consumed $50 \mathrm{~mL}$ of either OPO or HOSO, containing 357 and 420 ppm vitamin E, respectively, to meet the nutritional requirements of fat intake. Intake of macronutrients and micronutrients (vitamin E) was analyzed using the DIAL software (Faculty of Pharmacy, Complutense University of Madrid).

The results obtained showed that the average consumption of vitamin E increased from $9.61 \mathrm{mg} /$ day (intake of volunteers before starting the study) to $17.76 \mathrm{mg} /$ day and $18.45 \mathrm{mg}$ /day after the intervention with OPO and HOSO, respectively. Acceptance tests to evaluate the level of satisfaction of both oils showed higher preference of OPO compared to HOSO.

OPO was well accepted and, thus, constitutes an important dietary source of vitamin E covering the recommended daily intake. It is worth thinking about OPO as part of a healthy diet.

\section{Conflict of Interest}

This study has been funded by the Interprofesional del Aceite de Orujo de Oliva (ORIVA). 